\title{
Expression of SMARCD1 interacts with age in association with asthma control on inhaled corticosteroid therapy
}

Michael J. McGeachie ${ }^{1}$ D, Joanne E. Sordillo², Amber Dahlin', Alberta L. Wang ${ }^{1}$, Sharon M. Lutz², Kelan G. Tantisira', Ronald Panganiban ${ }^{3}$, Quan Lu ${ }^{3}$, Satria Sajuthi ${ }^{4}$, Cydney Urbanek ${ }^{4}$, Rachel Kelly ${ }^{1}$, Benjamin Saef ${ }^{4}$, Celeste Eng ${ }^{5}$, Sam S. Oh ${ }^{5}$, Alvin T. Kho ${ }^{6}$, Damien C. Croteau-Chonka ${ }^{1}$, Scott T. Weiss ${ }^{1}$, Benjamin A. Raby ${ }^{1,7}$, Angel C. Y. Mak ${ }^{4}$, Jose R. Rodriguez-Santana ${ }^{8}$, Esteban G. Burchard ${ }^{4}$, Max A. Seibold ${ }^{5}$ and Ann Chen Wu ${ }^{2^{*}}$

\begin{abstract}
Background: Global gene expression levels are known to be highly dependent upon gross demographic features including age, yet identification of age-related genomic indicators has yet to be comprehensively undertaken in a disease and treatment-specific context.

Methods: We used gene expression data from CD4+ lymphocytes in the Asthma BioRepository for Integrative Genomic Exploration (Asthma BRIDGE), an open-access collection of subjects participating in genetic studies of asthma with available gene expression data. Replication population participants were Puerto Rico islanders recruited as part of the ongoing Genes environments \& Admixture in Latino Americans (GALA II), who provided nasal brushings for transcript sequencing. The main outcome measure was chronic asthma control as derived by questionnaires. Genomic associations were performed using regression of chronic asthma control score on gene expression with age in years as a covariate, including a multiplicative interaction term for gene expression times age.
\end{abstract}

Results: The SMARCD1 gene (SWI/SNF-related matrix-associated actin-dependent regulator of chromatin subfamily D member 1) interacted with age to influence chronic asthma control on inhaled corticosteroids, with a doubling of expression leading to an increase of 1.3 units of chronic asthma control per year $\left(95 \% \mathrm{Cl}[0.86,1.74], p=6 \times 10^{-9}\right)$, suggesting worsening asthma control with increasing age. This result replicated in GALA II $\left(p=3.8 \times 10^{-8}\right)$. Cellular assays confirmed the role of SMARCD1 in glucocorticoid response in airway epithelial cells.

Conclusion: Focusing on age-dependent factors may help identify novel indicators of asthma medication response. Age appears to modulate the effect of SMARCD1 on asthma control with inhaled corticosteroids.

\section{Background}

Asthma affects over 300 million persons globally and costs more than $\$ 50$ billion annually in the U.S. [1] Despite effective treatment options, exacerbations from asthma account for substantial preventable morbidity [2]. Most individuals with asthma respond to inhaled corticosteroids (ICS), the most effective asthma controller medication, with significant symptom improvement; however, approximately one third of individuals respond

\footnotetext{
* Correspondence: ann.wu@childrens.harvard.edu

${ }^{2}$ PRecisiOn Medicine Translational Research (PROMoTeR) Center, Department of Population Medicine, Harvard Pilgrim Health Care Institute and Harvard Medical School, 401 Park Drive, Suite 401, Boston, MA 02215-5301, USA Full list of author information is available at the end of the article
}

minimally or not at all [3]. Furthermore, age appears to modify ICS response, with increasing treatment failures for each year over the age of 30 [4]. While genetics explains substantial variability in asthma drug disposition and effects $[5,6]$, the field of pharmacogenomics has not addressed the role of age in modulating response to asthma medications.

Asthma pharmacogenomic studies to date have shed light on biological mechanisms. For example, a genomic study characterizing transcriptomes identified multiple genes involved in the inflammatory pathway that influence ICS response including Cysteine Rich Secretory Protein LCCL Domain Containing 2 (CRISPLD2) [7]. 
CRISPLD2 mRNA has been shown to strongly vary with age [8], and protein levels were shown to increase in response to treatment with a known pro-inflammatory cytokine, interleukin 1 beta (IL1 $\beta$ ). Moreover, a transcriptomic study of ORMDL Sphingolipid Biosynthesis Regulator 3 (ORMDL3) found that a variant in that gene might influence the route of anti-inflammatory action of glucocorticoids by modifying the transcriptional activation of ORMDL3 in subjects with asthma [9]. As ORMDL3 is associated with childhood-onset asthma, ORMDL3's influence on the inflammatory pathway may be age-dependent; however, no published studies have examined this [9].

Global gene expression levels are known to be highly dependent upon gross demographic features including age $[10,11]$, yet identification of age-related genomic indicators has yet to be comprehensively undertaken in an asthma-specific context. The objective of this study was to discover genomic indicators specific to response to ICS in individuals with asthma by accounting for agedependent genomic interactions.

\section{Methods}

\section{Populations}

We studied populations with available gene expression data on subjects with asthma and ICS use. Data from subjects in the Asthma BioRepository for Integrative Genomic Exploration (Asthma BRIDGE) were used in this study as the discovery population. Asthma BRIDGE participants are taken from the EVE network of asthma genetic studies [12]. In particular, the present analysis focused on ICS-using participants from the CARE [13, 14], CAG, and GRAAD [15] studies. These are described in greater detail in the Additional file 1. Secondary data analysis of these cohorts was approved by the Partners Healthcare IRB, approval number HL71392-2. The replication population included Puerto Rico islanders who were recruited as part of the ongoing Genes environments \& Admixture in Latino Americans (GALA II) study described elsewhere [16, 17]. Asthma was defined by a physician's diagnosis and the presence of 2 or more symptoms of coughing, wheezing, or shortness of breath in the 2 years before enrollment. Subjects who reported ICS use in the past year were included. The study was approved by local institutional review boards, and written assent/consent was received from all subjects and their parents.

\section{Outcomes}

We used a series of questions to assess asthma severity and asthma control during the 6 months preceding blood draw for the Asthma BRIDGE expression data. Eleven questions regarding asthma severity and control were combined into a single aggregate Chronic Asthma
Control Score (CACS). These questions were based on the Asthma Control Questionnaire [18] and the Asthma Control Test [19] questions, and are detailed in the Additional file 1. The CACS score ranged from 0 (excellent asthma control) to 44 (very poor asthma control).

In GALA II, the Childhood Asthma Control Test (C$\mathrm{ACT}$ ) and the Asthma Control Questionnaire (ACQ) [18] were used to derive a measure of asthma control. Both the C-ACT and ACQ measure how well-controlled a subject's asthma is and can detect any changes in their control, due to either spontaneous occurrence or resulting from treatment. Each questionnaire consists of 5 domains: nighttime symptoms, daytime symptoms, activity limitations, use of rescue medication, and lung function. The five domains were each categorized into three levels of control: controlled (0), not well controlled (1), or very poorly controlled (2). The asthma control was determined to be the maximum value across the five domains, with higher values indicating worse control. Complete details are in Additional file 1: Table S2. In this analysis, only subjects taking ICS in the previous year were included, and the cohort was dichotomized into the controlled group ( 0$)$ and the poorly-controlled group (1 or 2).

\section{Expression data}

We used mRNA expression data from CD4+ lymphocytes from the Asthma BioRepository for Integrative Genomic Exploration (Asthma BRIDGE). This data was previously processed on Illumina Human HT-12 v4 arrays, according to manufacturers' protocol (Illumina, San Diego CA). This array assays 47,036 different mRNA features. These data were processed through quality control metrics at Brigham and Women's Hospital, as described previously [20]. Asthma BRIDGE contains gene expression data on several different cell types, and for greatest power, we chose the CD4+ lymphocytes as an asthma-relevant cell type that contains the highest number of samples spanning an adult age-range. These expression data have been previously described [20].

The GALA II expression data were obtained using methods for nasal epithelial cell collection and processing developed in collaboration with the National Institutes of Health/National Institute of Allergy and Infectious Diseases-sponsored Inner City Asthma Consortium, optimizing for collection and confirmation of columnar epithelial cell type, RNA yield, and specimen-collector training [21]. Briefly, nasal epithelial cells were collected from behind the inferior turbinate with a cytology brush using a nasal illuminator. The collected brush was submerged in RLT Plus lysis buffer plus $\beta$-mercaptoethanol and frozen at $-80^{\circ} \mathrm{C}$ until extraction. mRNA in samples were then quantified using RNA-seq. The total read count of SMARCD1 was size factor-normalized followed by variance-stabilized 
transformation using the software package DESeq2 [22] prior to modeling.

\section{Statistical analysis}

Clinical and demographic data were compared across cohort subdivisions using analysis of variance (ANOVA) for continuous outcomes and chi square tests for discrete outcomes (Table 1).

Genomic associations were performed using linear regression of CACS on log base 2 transformation of gene expression with age in years as a covariate, including a multiplicative interaction term for gene expression times age. This interaction term was of principal interest to the current study. Significance of association was assessed by linear regression test using a significance threshold of $<0.05$ after Bonferroni correction. All operations were performed in MATLAB R2018a (MathWorks, Natick, MA).

Replication of the SMARCD1 interaction with age in GALA II was conducted with multinomial logistic regression analysis using multinom $\mathrm{R}$ package with the asthma control category as the outcome with the wellcontrolled group as the baseline.

Individual top age times gene expression interaction hits were interrogated using NDEX, a publically available catalogue of biological networks [23].

\section{In vitro verification}

A549/NF-kB-luc reporter cells were transfected with either scramble control or one of two SMARCD1 small interfering RNAs (siRNA). These cells are responsive to IL- $1 \beta$ stimulation, which is reduced by dexamethasone, a glucocorticoid receptor agonist, indicating that these cells exhibit glucocorticoid-mediated tethered transrepression of NF- $\mathrm{KB}$ [24]. $48 \mathrm{~h}$ post-transfection, the cells were stimulated with $5 \mathrm{ng} / \mathrm{mL}$ IL- $1 \beta \pm 5 \mathrm{nM}$ Dexamethasone. Luciferase assays were performed after $18 \mathrm{~h}$ treatment. The luciferase activity was normalized to its own untreated scramble control. The luciferase activities of cells treated with IL-1 $1 \beta+$ Dex and transfected with siRNAs were calculated and compared to that of cells treated with IL-1 $\beta+$ Dex and transfected with scramble control. All experimental conditions were completed in three replicates $(n=3)$.

\section{Results}

There were 118 subjects with gene expression data passing quality control from CD4+ lymphocytes in Asthma BRIDGE who reported taking inhaled corticosteroids as a controller medication in the previous year. These subjects had mean Chronic Asthma Control Scores (CACS) of $10.8(+/-6.9)$, shown in Fig. 1. A general trend emerged showing increasing CACS with increasing age (increase of $0.15 /$ year, $95 \%$ confidence interval [0.079, 0.22 ], $p=3.4 \times 10^{-5}$ ), indicating worsening asthma control with advancing age. This trend remained significant when stratified by sex, and no significant difference was observed in males (increase of $0.17 /$ year, $95 \%$ CI [0.065, 0.28 ], $p=0.0012$ ) versus females (increase of $0.12 /$ year, 95\% CI [0.011, 0.22], $p=0.03$ ).

In Asthma BRIDGE, the SMARCD1 gene (SWI/SNFrelated matrix-associated actin-dependent regulator of chromatin subfamily D member 1) strongly interacted with age to indicate higher CACS on inhaled corticosteroids, with a doubling of expression associated with an

Table 1 Cohorts included in Asthma BRIDGE. Continuous variables are reported as means with (+/- standard deviation). Binary variables are reported as counts with (percentage of cohort)

\begin{tabular}{|c|c|c|c|c|}
\hline & GRAAD & CAG & CARE & $p$-value \\
\hline $\bar{N}$ & 35 & 25 & 58 & \\
\hline CACS & $13.97(+/-8.01)$ & $12.83(+/-6.38)$ & $8.12(+/-5.16)$ & $5.70 \mathrm{E}-05$ \\
\hline Age & $42.94(+/-12.70)$ & $38.52(+/-13.35)$ & $13.49(+/-3.45)$ & 1.30E-29 \\
\hline Sex (\# Male) & $9(25.71 \%)$ & $6(24.00 \%)$ & $40(68.97 \%)$ & 1.10E-05 \\
\hline \multicolumn{5}{|l|}{ Race } \\
\hline Non-Hispanic White & $0(0.00 \%)$ & $4(16.00 \%)$ & $35(60.34 \%)$ & 2.00E-09 \\
\hline African American & $30(85.71 \%)$ & $20(80.00 \%)$ & $8(13.79 \%)$ & $3.70 \mathrm{E}-13$ \\
\hline Hispanic & $0(0.00 \%)$ & $0(0.00 \%)$ & $9(15.52 \%)$ & 0.0065 \\
\hline Other/Mixed & $5(14.29 \%)$ & $1(4.00 \%)$ & $6(10.34 \%)$ & 0.43 \\
\hline Age Asthma Onset & $18.25(+/-16.22)$ & $5.73(+/-7.79)$ & $2.16(+/-2.09)$ & 1.50E-11 \\
\hline Atopy & $12(34.29 \%)$ & $9(36.00 \%)$ & $34(58.62 \%)$ & 0.036 \\
\hline Intra-Uterine Smoke & $5(14.29 \%)$ & $5(20.00 \%)$ & $3(5.17 \%)$ & 0.11 \\
\hline Environmental Smoke Exposure & $21(60.00 \%)$ & $12(48.00 \%)$ & $9(15.52 \%)$ & 2.80E-05 \\
\hline Ever Smoker & $13(37.14 \%)$ & $3(12.00 \%)$ & $0(0.00 \%)$ & 2.60E-06 \\
\hline
\end{tabular}

$P$-values for continuous outcomes are from ANOVA, binary outcomes are from chi-squared tests. CACS: Chronic Asthma Control Score. CARE: Childhood Asthma Research and Education study. CAG: Chicago Asthma Genetics study. GRAAD: Genomic Research on Asthma in the African Diaspora 


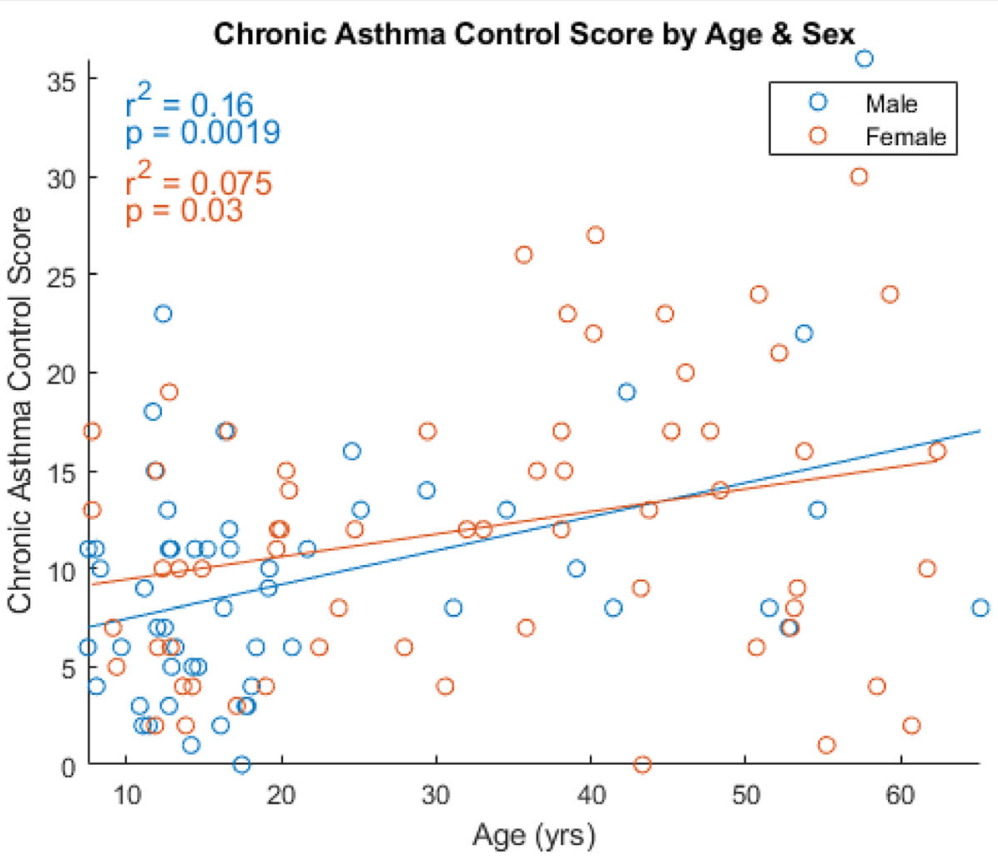

Fig. 1 Chronic Asthma Control Score (CACS) by Age, stratified by sex. Shown are trend lines along with the distribution of CACS by age. Both trendlines are significant

increase of 1.3 units of CACS per year increase in age (95\% CI $\left.[0.86,1.74], p=6 \times 10^{-9}\right)$, indicating increasing importance of SMARCD1 with advancing age (Fig. 2). In the replication population, GALA II, the age-bySMARCD1 interaction was associated with poorlycontrolled asthma compared to subjects with controlled asthma, with a ratio of odds ratios relative to control of $1.72\left(95 \%\right.$ CI $\left.[1.42,2.10], p=3.8 \times 10^{-8}\right)$, indicating increasing probability of being in the poorly-controlled group with age.

We observed that both the specific study within Asthma BRIDGE and self-identified race were strongly



Fig. 2 Blue: Asthma BRIDGE participants younger than 30 years; Red: Asthma BRIDGE participants 31-50 years old; Yellow: Asthma BRIDGE participants 50 years or older. Differing slopes indicates interaction of SMARCD1 with Age on CACS 
split by age (Table 1): GRAAD and CAG are adult studies and CARE is a study of children. Similarly, CARE is predominantly non-Hispanic white with a minority population of Hispanic white; while GRAAD and CAG are mostly African American. Since we wanted to focus on the interaction between gene expression and age in asthma control, we did not include either of these demographic variables in our main analysis. However, there was no significant association of race or study with $S M A R C D 1$ expression after adjustment for age (ANOVA, $p=0.29$ and $p=0.3$, respectively).

For completeness, we also considered SMARCD1-age interaction in other cell types available in ABRIDGE. These included (1) Alveolar Macrophages $(n=33, p=.75)$, although these samples did not span a similar age range (ages 8 to 22 years). (2) Bronchial Epithelium ( $n=16$, $p=.2)$, Bronchoalveolar cells $(n=12, p=.22)$, and whole blood ( $n=4$, p not computed), although these had low sample sizes. Finally (5) CD4+ lymphocytes stimulated with hemagglutinin $(n=139, p=.01)$, although this stimulation may not be representative of asthma biology.

To investigate whether SMARCD1 plays a role in the inflammatory response as well as in the anti-inflammatory action of corticosteroids in vitro, we knocked down SMARCD1 in a human lung epithelial cell line (similar to the replication samples which were enriched for airway epithelium) stably expressing nuclear factor-kappa B (NF$\kappa B)$ luciferase reporter [24]. We transfected this reporter cell line with two different SMARCD1 siRNAs. One of these resulted in a large (greater than $80 \%$ ) reduction of SMARCD1 expression relative to scramble-control siRNA transfection (Fig. 3). In this successful knockdown of SMARCD1, we observed a significant increase in luciferase activity relative to scramble-control in response to both IL-1 $\beta$ stimulation $(p<0.01)$ and IL-1 $\beta$ plus dexamethasone $(p<0.01)$ (Fig. 4). This verified that, at least in vitro,

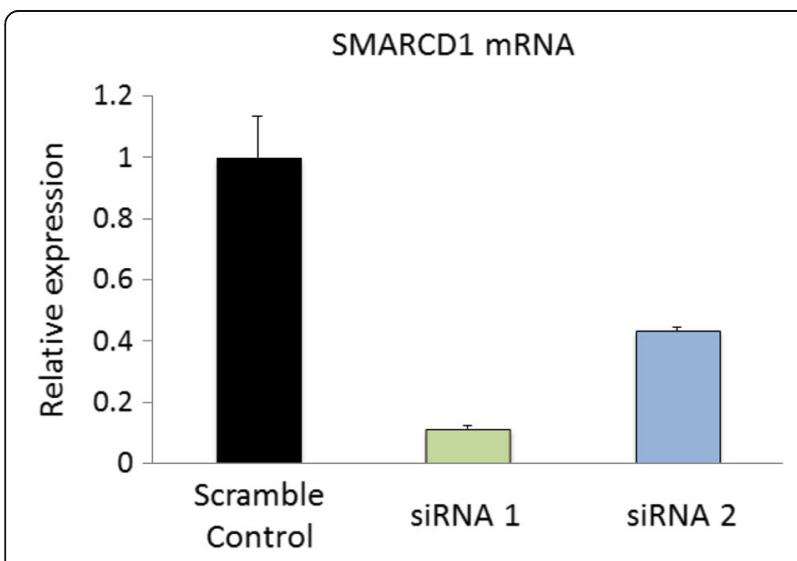

Fig. 3 Relative expression of SMARCD1 in A549 cells after transfection with two separate short interfering RNAs (siRNA). siRNA 1 showed over $80 \%$ reduction of SMARCD1 expression compared to transfection with a scramble RNA control
SMARCD1 is an important part of the inflammatory response and its absence increases the anti-inflammatory action of corticosteroid treatment.

In addition to performing pathway enrichment studies using our own results (see Additional file 1), we also investigated connections of the SMARCD1 gene and its protein product (also labeled SMARCD1) in NDEX, a searchable collection of gene expression and proteinprotein interaction networks from multiple network and pathway databases [23]. A search on SMARCD1 revealed the following biologically relevant network for our phenotype of interest, ICS response, in the current analysis: The glucocorticoid receptor regulatory network (a protein-protein interaction network) was derived from the latest BioPAX3 version of the Pathway Interaction Database. A portion of the larger network including our gene of interest (the one-step adjacent network surrounding SMARCD1) is shown in Fig. 5. This diagram indicates that cortisol is a controller of $S M A R C D 1$, and that expression of SMARCD1 is related to SGK1 (glucocorticoid regulated kinase 1) the glucocorticoid receptor Nuclear Receptor Subfamily 3 Group C Member 1 (NR3C1).

\section{Discussion}

Our study has three key findings. First, age appears to modulate the association of SMARCD1 with chronic asthma control. Secondly, focusing on age-dependent factors is likely to yield important indicators of asthma medication response. Third, interactions between age and expression of SMARCD1 in CD4+ lymphocytes were replicated in nasal samples enriched for respiratory epithelial cells, suggesting that the potential age-dependent effects of SMARCD1 on asthma control could be mediated by systemic as well as locally induced changes in the airways. That SMARCD1 interacts with age to affect asthma control on ICS, across two different cell types, in two different racial ancestry groups, and using two different measures of asthma control, indicates a robust effect.

SMARCD1, a member of the SWI/SNF chromatin remodeling complex family, regulates gene transcription by binding specific transcriptional factors and altering local chromatin structure [25]. To our knowledge, SMARCD1 has not been reported as associated with ICS response in prior studies, although it has been found to be associated with asthma in a prior analysis that used GEO data from adult cohorts of the Unbiased Biomarkers for the Predictions of Respiratory Disease Outcomes (UBIOPRED) research study [26]. Protein-protein interaction network data support the role of SMARCD1 in modulating asthma control while on ICS. Synthetic glucocorticoid medications (such as inhaled budesonide and dexamethasone) are potent inducers of glucocorticoid signaling, 


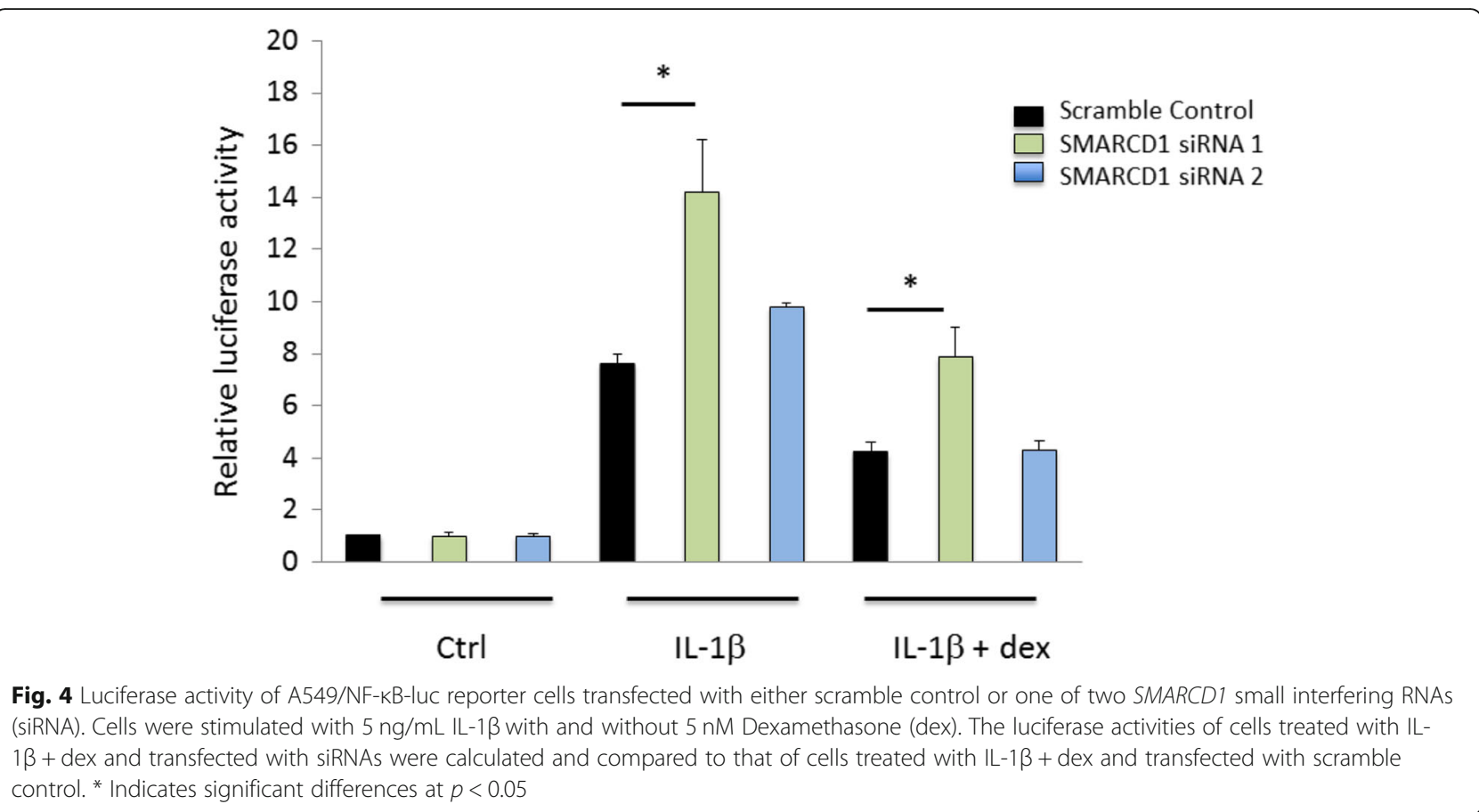



Fig. 5 SMARCD1 nearest-neighbor adjacency subnetwork with the glucocorticoid receptor response pathway. The orange arrow originating from cortisol to SMARCD1 indicates that cortisol is a controller of SMARCD1. The blue directed edge from SMARCD1 to SGK1 (glucocorticoid regulated kinase 1) demonstrates the known relationship between SMARCD1 expression and increased expression of SGK1. The gray dotted lines indicate that SMARCD1 co-complexes with the connected node proteins, including Nuclear Receptor Subfamily 3 Group C Member 1 (NR3C1), a glucocorticoid receptor exerting their anti-inflammatory effects through glucocorticoid receptor binding [27]. A search of NDEX [23] shows $S M A R C D 1$ as a key effector and regulatory protein within the glucocorticoid receptor regulatory network. In protein-protein interaction studies, SMARCD1 complexes with NRC31 (nuclear receptor subfamily 3 group C member 1), a glucocorticoid receptor [28]. We further validated the in vitro role of SMARCD1 in the inflammatory process and the action of dexamethasone on that process.

In addition to its direct involvement in glucocorticoid signaling, SMARCD1 is also associated with apoptosis pathways [29, 30]. Interestingly, in vitro studies of ICS response have identified apoptosis as a potential protective mechanism associated with resolution of asthmatic inflammation [31-34]. An analysis of gene regulatory networks for ICS response in mid-childhood identified enrichment of pro-apoptosis pathways in good ICS responders, and anti-apoptosis pathway enrichment in poor ICS responders. While cellular apoptosis is a natural process occurring at all stages of human development, changes in expression of apoptosis genes occur with age $[35,36]$. In our study, alterations in SMARCD1 expression with age may relate to its involvement in apoptosis pathways. Future studies will be required to understand whether age-associated changes in apoptosis pathways underlie the age-related effects of SMARCD1 on ICS response in asthmatics.

Our study has several strengths. Our gene expression analysis was appropriately timed to recent inhaled corticosteroid use. In our discovery analysis, asthma control while on ICS was assessed using a questionnaire based 
on the ACT criteria, which accounts for multiple aspects of control, including symptoms, quality of life metrics (missed school/work), medication use and health care utilization (emergency room/doctor visits for asthma). In addition to replication across tissue types, we found replication of the SMARCD1 by age interaction across multiple racial ethnic groups, which contributes to the generalizability of our findings. Our study also had some limitations. Assessment of asthma control was somewhat different in the discovery and replication cohorts, which may have affected our ability to replicate additional age-by-gene expression interactions in models of asthma control while on ICS. Although our in vitro validation demonstrated a biological relationship between SMARCD1, NF-kB, and dexamethasone, the nature of the experiment with cell lines precludes the assessment of age; thus this result does not directly support a differential effect of SMARCD1 with increasing age. Furthermore, this effect was in the opposite direction as one might expect: SMARCD1 knockdown increased NF-kB, which generally increases inflammation, which generally increases asthma symptoms, and would lead to higher CAC. We speculate that this difference in effect direction could be due to differences between in vitro and in vivo conditions, the inability to include age in the cell lines, or differences between airway epithelial cells and CD4+ lymphocytes. However, our strongest evidence indicates that increasing SMARCD1 is associated with worsening asthma control.

\section{Conclusions}

In conclusion, our study provides evidence that focusing on age-dependent factors may help identify novel indicators of asthma medication response. Age appears to modulate the association of SMARCD1 with asthma control in subjects taking inhaled corticosteroids.

\section{Supplementary information}

Supplementary information accompanies this paper at https://doi.org/10. 1186/s12931-020-1295-4.

Additional file 1. Supplementary Methods, Results, Discussion, and Tables.

\footnotetext{
Abbreviations

ACQ: Asthma Control Questionnaire; ANOVA: Analysis of Variance; Asthma BRIDGE: Asthma BioRepository for Integrative Genomic Exploration; CACS: Chronic Asthma Control Score; C-ACT: Childhood Asthma Control Test; CAG: Chicago Asthma Genetics study; CARE: Childhood Asthma Research and Education; CRISPLD2: Cysteine Rich Secretory Protein LCCL Domain Containing 2; GALA II: Genes environments \& Admixture in Latino Americans II; GRAAD: Genomic Research on Asthma in the African Diaspora; ICS: Inhaled Corticosteroids; mRNA: Messenger RNA; messenger Ribonucleic Acid; NR3C1: Nuclear Receptor Subfamily 3 Group C Member 1; ORMDL3: ORMDL Sphingolipid Biosynthesis Regulator 3; SGK1: Serine/Threonine-Protein Kinase; siRNAs: Small interfering RNA; SMARCD1: SWI/SNF-related matrix-associated actin-dependent regulator of chromatin subfamily $D$ member 1 ; $U$ -
}

BIOPRED: Unbiased Biomarkers for the Predictions of Respiratory Disease Outcomes

\section{Acknowledgements}

Not applicable.

\section{Authors' contributions}

MJM and ACW contributed substantially to the study design, analysis, interpretation, funding, and writing of the manuscript. JES, AD, ALW, SML, RP, $\mathrm{QL}, \mathrm{SS}, \mathrm{CU}, \mathrm{RK}, \mathrm{BS}, \mathrm{CE}, \mathrm{SSO}, \mathrm{ATK}$, and DCCC contributed substantially to the study design, analysis, interpretation and writing of the manuscript. KGT, STW, BAR, ACYM, JRRS, EGB, and MAS contributed substantially to the study design, funding, interpretation, and critical revisions of the manuscript. All authors read and approved the final manuscript.

\section{Funding}

Funding was provided by National Institutes of Health (NIH) grants: R01HL118455, and RC2 HL101543. R01 HD085993 (ACW), R00 HL109162 (JES), R01 HL139634 (MJM), K01 HL125858 (SML), K01 HL127265 (DCCC). Funding agencies had no input on the results, analysis, or reporting of data presented herein.

\section{Availability of data and materials}

Gene expression and phenotype data from ABRIDGE are publicly available to download from the Database of Genotypes and Phenotypes (dbGaP accession number pending) or from the Gene Expression Omnibus (GEO accession number GSE22324), respectively.

\section{Ethics approval and consent to participate}

The secondary data analysis of these cohorts conducted in this study was approved by the Partners Healthcare IRB, approval number HL-71392-2. GALA II was approved by local institutional review boards, and written assent/consent was received from all subjects and their parents.

\section{Consent for publication}

Not Applicable.

\section{Competing interests}

The authors declare that they have no competing interests.

\section{Author details}

${ }^{1}$ Channing Division of Network Medicine, Department of Medicine, Brigham and Women's Hospital and Harvard Medical School, Boston, MA, USA.

${ }^{2}$ PRecisiOn Medicine Translational Research (PROMoTeR) Center, Department of Population Medicine, Harvard Pilgrim Health Care Institute and Harvard Medical School, 401 Park Drive, Suite 401, Boston, MA 02215-5301, USA. ${ }^{3}$ Program in Molecular and Integrative Physiological Sciences, Departments of Environmental Health and Genetics \& Complex Diseases, Harvard T.H. Chan School of Public Health, Boston, MA, USA. ${ }^{4}$ Center for Genes, Environment and Health, Department of Pediatrics, National Jewish Health, Denver, CO, USA. ${ }^{5}$ Department of Medicine, University of California San Francisco, San Francisco, CA, USA. ${ }^{6}$ Computational Health Informatics Program, Boston Children's Hospital, Boston, MA, USA. ${ }^{7}$ Division of Pulmonary and Respiratory Diseases, Boston Children's Hospital, Boston, MA, USA. ${ }^{8}$ Centro de Neumologia Pediatrica, San Juan, PR, USA.

Received: 2 October 2019 Accepted: 14 January 2020 Published online: 28 January 2020

\section{References}

1. O'Brien S. Statistics: Asthma.net; 2016 Available from: https://asthma.net/ basics/statistics/.

2. Gruffydd-Jones K. Unmet needs in asthma. Ther Clin Risk Manag. 2019;15: 409-21.

3. Chan MT, Leung DY, Szefler SJ, Spahn JD. Difficult-to-control asthma: clinical characteristics of steroid-insensitive asthma. J Allergy Clin Immunol. 1998; 101(5):594-601.

4. Dunn RM, Lehman E, Chinchilli VM, Martin RJ, Boushey HA, Israel E, et al. Impact of age and sex on response to asthma therapy. Am J Respir Crit Care Med. 2015;192(5):551-8. 
5. Tantisira KG, Lasky-Su J, Harada M, Murphy A, Litonjua AA, Himes BE, et al. Genomewide association between GLCCl1 and response to glucocorticoid therapy in asthma. N Engl J Med. 2011;365(13):1173-83.

6. Ortega VE, Meyers DA, Bleecker ER. Asthma pharmacogenetics and the development of genetic profiles for personalized medicine. Pharmgenomics Pers Med. 2015:8:9-22.

7. Himes BE, Jiang X, Wagner P, Hu R, Wang Q, Klanderman B, et al. RNA-Seq transcriptome profiling identifies CRISPLD2 as a glucocorticoid responsive gene that modulates cytokine function in airway smooth muscle cells. PLoS One. 2014;9(6):e99625

8. Peters MJ, Joehanes R, Pilling LC, Schurmann C, Conneely KN, Powell J, et al. The transcriptional landscape of age in human peripheral blood. Nat Commun. 2015;6:8570.

9. Berce $V$, Kozmus CE, Potocnik U. Association among ORMDL3 gene expression, 17q21 polymorphism and response to treatment with inhaled corticosteroids in children with asthma. Pharmacogenomics J. 2013;13(6):523-9.

10. Vinuela A, Brown AA, Buil A, Tsai PC, Davies MN, Bell JT, et al. Agedependent changes in mean and variance of gene expression across tissues in a twin cohort. Hum Mol Genet. 2018;27(4):732-41.

11. Glass D, Vinuela A, Davies MN, Ramasamy A, Parts L, Knowles D, et al. Gene expression changes with age in skin, adipose tissue, blood and brain. Genome Biol. 2013;14(7):R75.

12. Torgerson DG, Ampleford EJ, Chiu GY, Gauderman WJ, Gignoux CR, Graves PE, et al. Meta-analysis of genome-wide association studies of asthma in ethnically diverse north American populations. Nat Genet. 2011;43(9):887-92.

13. Guilbert TW, Morgan WJ, Krawiec M, Lemanske RF Jr, Sorkness C, Szefler SJ, et al. The prevention of early asthma in kids study: design, rationale and methods for the childhood asthma research and education network. Control Clin Trials. 2004;25(3):286-310.

14. Denlinger LC, Sorkness CA, Chinchilli VM, Lemanske RF Jr. Guidelinedefining asthma clinical trials of the National Heart, Lung, and Blood Institute's asthma clinical research network and childhood asthma research and education network. J Allergy Clin Immunol. 2007;119(1):3-11 quiz 2-3.

15. Mathias RA, Grant AV, Rafaels N, Hand T, Gao L, Vergara C, et al. A genomewide association study on African-ancestry populations for asthma. J Allergy Clin Immunol. 2010;125(2):336-46 e4.

16. Kumar R, Nguyen EA, Roth LA, Oh SS, Gignoux CR, Huntsman S, et al. Factors associated with degree of atopy in Latino children in a nationwide pediatric sample: the genes-environments and admixture in Latino asthmatics (GALA II) study. J Allergy Clin Immunol. 2013;132(4):896-905 e1.

17. Borrell LN, Nguyen EA, Roth LA, Oh SS, Tcheurekdjian H, Sen S, et al. Childhood obesity and asthma control in the GALA II and SAGE II studies. Am J Respir Crit Care Med. 2013;187(7):697-702.

18. Juniper EF, O'Byrne PM, Guyatt GH, Ferrie PJ, King DR. Development and validation of a questionnaire to measure asthma control. Eur Respir J. 1999; 14(4):902-7.

19. Nathan RA, Sorkness CA, Kosinski M, Schatz M, Li JT, Marcus P, et al. Development of the asthma control test: a survey for assessing asthma control. J Allergy Clin Immunol. 2004;113(1):59-65.

20. Croteau-Chonka DC, Qiu W, Martinez FD, Strunk RC, Lemanske RF Jr, Liu AH, et al. Gene expression profiling in blood provides reproducible molecular insights into asthma control. Am J Respir Crit Care Med. 2017;195(2):179-88.

21. Poole A, Urbanek C, Eng C, Schageman J, Jacobson S, O'Connor BP, et al. Dissecting childhood asthma with nasal transcriptomics distinguishes subphenotypes of disease. J Allergy Clin Immunol. 2014;133(3):670-8.e12.

22. Love MI, Huber W, Anders S. Moderated estimation of fold change and dispersion for RNA-seq data with DESeq2. Genome Biol. 2014;15(12):550.

23. Pillich RT, Chen J, Rynkov V, Welker D, Pratt D. NDEx: a community resource for sharing and publishing of biological networks. Methods Mol Biol. 2017; 1558:271-301.

24. Jiang X, Dahlin A, Weiss ST, Tantisira K, Lu Q. A high-throughput chemical screen identifies novel inhibitors and enhancers of anti-inflammatory functions of the glucocorticoid receptor. Sci Rep. 2017:7(1):7405.

25. Zhang $P$, Li L, Bao Z, Huang F. Role of BAF60a/BAF60c in chromatin remodeling and hepatic lipid metabolism. Nutr Metab (Lond). 2016;13:30.

26. Enguita DM. Identification of personalized multi-omic disease modules in asthma. Skövde, Sweden: University of Skövde; 2018.

27. Gras D, Chanez P, Vachier I, Petit A, Bourdin A. Bronchial epithelium as a target for innovative treatments in asthma. Pharmacol Ther. 2013;140(3): 290-305.
28. Vitellius G, Fagart J, Delemer B, Amazit L, Ramos N, Bouligand J, et al. Three novel heterozygous point mutations of NR3C1 causing glucocorticoid resistance. Hum Mutat. 2016;37(8):794-803.

29. Hong CF, Lin SY, Chou YT, Wu CW. MicroRNA-7 compromises p53 proteindependent apoptosis by controlling the expression of the chromatin remodeling factor SMARCD1. J Biol Chem. 2016;291(4):1877-89.

30. Li J, Zhou Q, Yang T, Li Y, Zhang Y, Wang J, et al. SGK1 inhibits PM2.5induced apoptosis and oxidative stress in human lung alveolar epithelial A549cells. Biochem Biophys Res Commun. 2018;496(4):1291-5.

31. Uddin M, Nong G, Ward J, Seumois G, Prince LR, Wilson SJ, et al. Prosurvival activity for airway neutrophils in severe asthma. Thorax. 2010;65(8):684-9.

32. Vazquez-Tello A, Halwani R, Hamid Q, Al-Muhsen S. Glucocorticoid receptorbeta up-regulation and steroid resistance induction by IL-17 and IL-23 cytokine stimulation in peripheral mononuclear cells. J Clin Immunol. 2013; 33(2):466-78.

33. White SR, Dorscheid DR. Corticosteroid-induced apoptosis of airway epithelium: a potential mechanism for chronic airway epithelial damage in asthma. Chest. 2002;122(6 Suppl):278S-84S.

34. Melis M, Siena L, Pace E, Gjomarkaj M, Profita M, Pirazzoli A, et al. Fluticasone induces apoptosis in peripheral T-lymphocytes: a comparison between asthmatic and normal subjects. Eur Respir J. 2002;19(2):257-66.

35. Chaves CF, Mazzotti DR, Cendoroglo MS, Ramos LR, Tufik S, Silva VCD, et al. Genes related to maintenance of autophagy and successful aging. Arq Neuropsiquiatr. 2018;76(12):831-9.

36. Rufini A, Tucci P, Celardo I, Melino G. Senescence and aging: the critical roles of p53. Oncogene. 2013;32(43):5129-43.

\section{Publisher's Note}

Springer Nature remains neutral with regard to jurisdictional claims in published maps and institutional affiliations.

Ready to submit your research? Choose BMC and benefit from:

- fast, convenient online submission

- thorough peer review by experienced researchers in your field

- rapid publication on acceptance

- support for research data, including large and complex data types

- gold Open Access which fosters wider collaboration and increased citations

- maximum visibility for your research: over $100 \mathrm{M}$ website views per year

At $\mathrm{BMC}$, research is always in progress.

Learn more biomedcentral.com/submissions 